\title{
Das Medusenhaupt in Mythos und Medizin - Versuch einer ganzheitlichen Betrachtung
}

\section{The Head of Medusa in Mythology and Medicine - Attempt at a Holistic Approach}

\section{Bibliografie}

DOI $10.1055 / \mathrm{s}-2007-966542$

Online-Publikation: 23.7. 2007

Akt Dermatol 2007; 33:

388-393 @ Georg Thieme

Verlag KG Stuttgart · New York ISSN 0340-2541

\section{Korrespondenzadresse} Dr. med. Rudolf Möhn Arzt für Hautkrankheiten und Allergologie Philippsruher Allee 35 63454 Hanau RMoehn@aol.com

\section{Zusammenfassung \\ $\nabla$}

Die Darstellung von Schlangenhaaren auf einem mittelalterlichen Kapitell war der Anlass, Beziehungen zum caput medusae der griechischen Mythologie und zu dem in der medizinischen Terminologie ebenfalls mindestens dreimal vorkommenden Begriff herzustellen. Als besonders eng erwies sich eine Korrelation zur caput-medusae-Variante des aus der Medizinhistorie be-

\section{Einleitung \\ $\nabla$}

Ein Besuch der in der ersten Hälfte des 13. Jahrhunderts anstelle eines 1143 gegründeten Prämonstratenserklosters entstandenen romanischen Basilika St. Johannes der Täufer in Frielendorf-Spieskappel (Schwalm-Ederkreis) hat mich zu dieser Arbeit angeregt. Besonders sehenswert sind die Kapitelle der an der westlichen Nordseite erhalten gebliebenen drei Säulen mit ihrem kräftigen plastischen Schmuck, von denen das mit der Darstellung von Männer- und Frauenköpfen, deren Bart- und Kopfhaare stark ineinander verflochten sind, besondere Aufmerksamkeit verdient ( Abb.1). Die schrecklichen Schlangenköpfe stammen zwar aus dem Barthaar des männlichen Kopfes, sind aber funktionell eher den weiblichen Figuren zuzuordnen, da sie dort den heiklen Brustbereich bedecken. Da auch das weibliche Haar in den männlichen Bart übergeht, soll jedenfalls extreme Verstrickung und Verfilzung ausgedrückt werden. Auf die merkwürdigen Blattornamente (?), die dem männlichen Mund entwachsen, soll hier nicht eingegangen werden. kannten Weichselzopfes, speziell in seiner artefiziellen Form, der ebenso wie das antike Vorbild Medusenhaupt magisch-mystische Qualität hat. Es wird versucht, mit Hilfe der Lehre C. G. Jungs vom „Kollektiven Unbewussten“ und dem darin verankerten Ursymbol Schlange eine gemeinsame Brücke zur ganzheitlichen Betrachtung für alle Manifestationen des caput medusae in Mythos, Kunst, Kultur, Biologie und Medizin zu bauen.

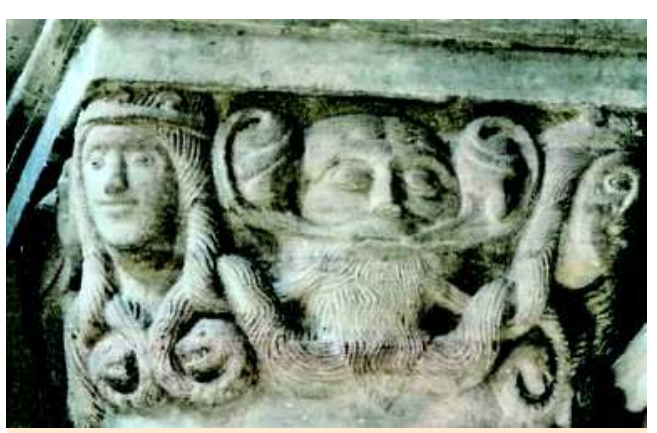

Abb. 1 Schlangenhaar von einem Kapitell in der Kirche zu Frielendorf-Spieskappel, Schwalm-Eder-Kreis (nach Führer zur Klosterkirche Spieskappel, Verlag Evangelischer Medienverband Kassel, 2006: 15).

\section{Beziehungen mittelalterlichen Schlangenhaares zum antiken Medusenhaupt $\nabla$}

Die Bildhauerarbeit ist von geradezu archaischer Ausdrucksgewalt, die tief in die Seele eindringt und auch beim modernen Menschen noch lähmende Angst und versteinerndes Entsetzen verbreiten kann. Jost [1] schreibt dazu, dass in den Westteilen (Abendseiten) romanischer Kirchen häufig Symbole des Bösen und Bedrohlichen dargestellt sind. In diesem Falle müsse an die Verstrickung von Adam und Eva gedacht werden, die im Paradies den Verlockungen der bösen 


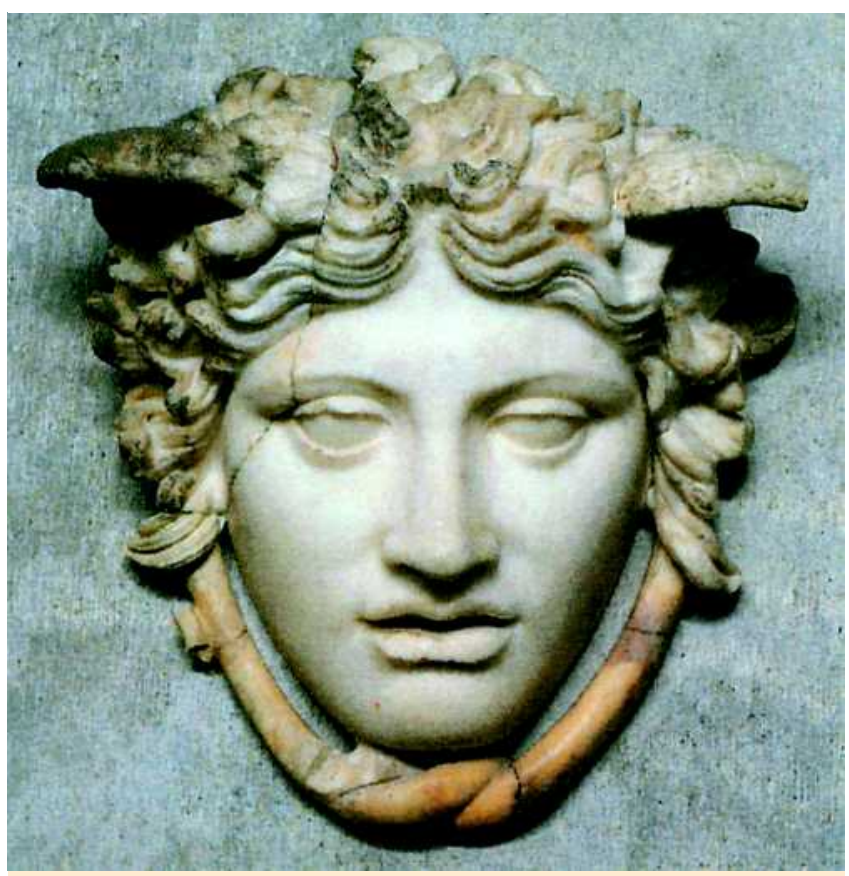

Abb. 2 Medusa Rondanini, römische Kopie nach dem Medusenhaupt auf dem Schild der Athenastatue im Parthenon (um 440 v. Chr.), München, Glyptothek.

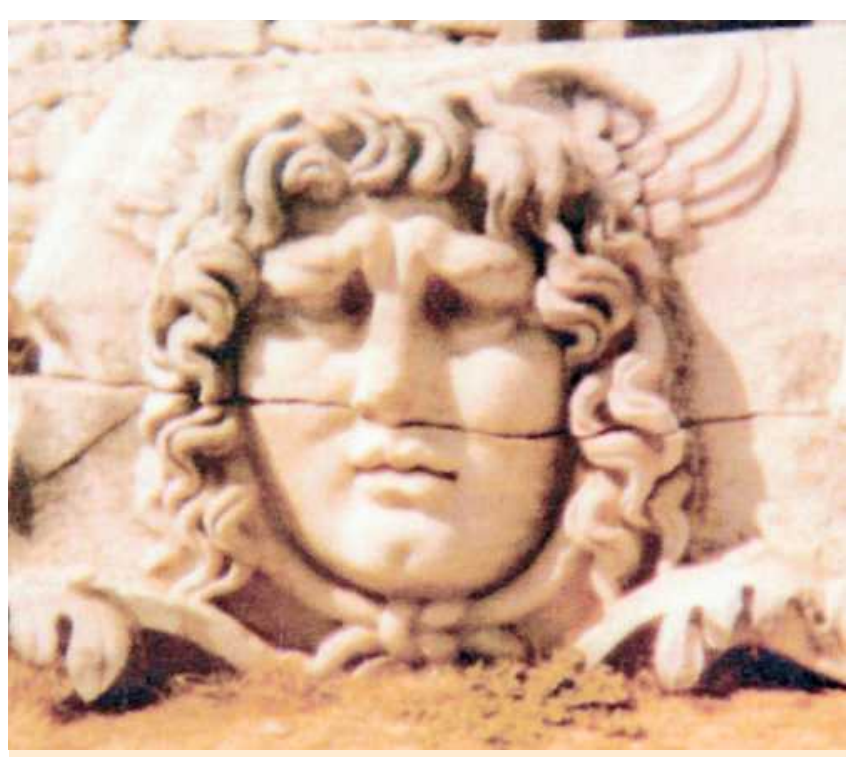

Abb. 3 Medusenhaupt vom Tempel zu Didyma, Westanatolien, späthellenistisch (Foto: Autor).

Schlange erlegen sind. Er weist darüber hinaus auch auf die Vielschichtigkeit der Schlangensymbolik überhaupt hin, betont aber, dass es letztlich wohl unklar bleiben müsse, was der Steinmetz habe ausdrücken wollen.

Zur Beantwortung dieser Frage sind nicht nur Theologen, Kunsthistoriker und Psychologen, sondern auch, wie sich zeigen wird, medizinhistorisch interessierte Ärzte, speziell Dermatologen, aufgerufen. Haar und Schlangen erinnern ganz spontan an das aus der griechischen Mythologie bekannte Gorgonen-, Medusen- oder auch Furienhaupt: Das schreckliche Ungeheuer Gorgon (gr.: die starr Blickende) wohnte nach Homer in der Unterwelt, später hat die Mythologie aus einem drei und diese zu Töchtern des Phorkys gemacht. Die schlimmste der drei Gorgo- nen mit den typischen Schlangenhäuptern war Medusa, zugleich aber als Einzige von ihnen sterblich. Perseus, der sagenhafte Sohn des Zeus und der Danae, wurde von seinem Stiefvater König Polydektes auf ein gefährliches Abenteuer geschickt, er sollte ihm nämlich den Kopf dieser Gorgon-Medusa bringen, dessen Anblick alle, die ihn ansahen, versteinerte. Mit Hilfe der Götter konnte sich Perseus aber dieses Kopfes bemächtigen, machte von der Möglichkeit der Versteinerung vieler Personen, die sich ihm in den Weg stellten, reichlich Gebrauch, und schenkte ihn dann der Göttin Athena, die ihn auf ihrem Brustpanzer anbrachte. Das Gorgonen- oder Medusenhaupt hat danach zahlreiche Künstler der Antike zu plastischen Darstellungen inspiriert. Die Frühesten zeigen ein rundes Gesicht mit aufgerissenen Augen, Raubtierzähnen und herausgestreckter Zunge, die Schlangenhaare kommen erst später und sind nur spärlich ausgeprägt. Die um 440 v. Chr. in klassischer Epoche entstandene Medusa Rondanini vom Schild der Athenastatue im Parthenon zu Athen, hier eine Kopie aus römischer Zeit ( $\bullet$ Abb. 2), zeigt zwar nach Goethe „das unsägliche Starren des Todes“, strahlt aber in ihrer zurückhaltenden Art fast noch „stille Einfalt, edle Größe“ (Winckelmann) aus und wirkt nicht ganz so furchterregend wie die von wilder Expressivität beherrschte späthellenistische Plastik in Didyma (Westanatolien) ( Abb.3). Beiden gemeinsam sind die zusätzlichen seitlichen flügelartigen Gebilde am Kopf, die wohl auf uralte griechische Kerasfrisuren (Hornfrisuren) zurückgehen, und die unter dem Kinn sich um den eigenen Schwanz ringelnde Schlange. Dieser „Uroboros“ ist auch in das Gedankengut der gnostischen Sekte der Ophiten (gr.: ophis, die Schlange) eingegangen, die u.a. eine solche „zyklische“ Schlange als das A und $\mathrm{O}$ (gr:: alpha und omega, die Anfangs- und Schlussbuchstaben des Alphabets) verehrten. Das $A$ und $O$ ist bis heute nach Offb. Joh. 22,13 auch in den Amtskirchen als Symbol für Jesus Christus gebräuchlich.

\section{Das archaische Ursymbol Schlange als verbindendes Glied zwischen den Zeiten $\nabla$}

Man wird einwenden, das Medusenhaupt entstamme einer ganz anderen Zeit und sei der heidnisch-griechischen Ikonografie zuzuordnen, die mit der mittelalterlich-christlichen, hier mit einer Variante in Spieskappel im Besonderen, nichts zu tun habe. Aber bereits vor der Renaissance hat es in der mittelalterlichen Kunst „Zitate“ aus der vorchristlichen, heidnischen Kunst gegeben, mit denen in der Regel das Hässliche, Böse, Niedrige und Grausame in Abgrenzung vom schönen und guten Christlichen charakterisiert werden sollte. „Das (Eindringen antiker Ikonografie, d.Verf.) wird um so wahrscheinlicher, als die Zeit um 1200, in der das Motiv (des Medusenhauptes, d. Verf.) in der mittelalterlichen Kunst zuerst auftaucht, in der Kunst ganz Europas eine Zeit allgemeinen Aufnehmens und Verarbeitens antiker Elemente und Anregungen war." (Michler) [2]. Bereits aus karolingischer Zeit, also gleichsam der Brückenzeit zwischen Antike und hohem Mittelalter, stammt beispielsweise auch die früher in Darmstadt befindliche Mölsheimer Fibel, die in der Mitte ein geflügeltes Medusenhaupt enthält. Darüber hinaus muss ganz allgemein gesagt werden, dass die Schlange ein archaisches, mit Angst und Schrecken besetztes Ursymbol ist, das in einer Art normativer Inversion aber auch wieder als Tier der Klugheit und Weisheit kultartig verehrt wird (u. a. Äskulapstab der Ärzte!). Es hat die Künstler aller Zeiten und Kulturen immer wieder inspiriert. Die Beispiele sind zahllos und können hier nicht alle behandelt werden. 


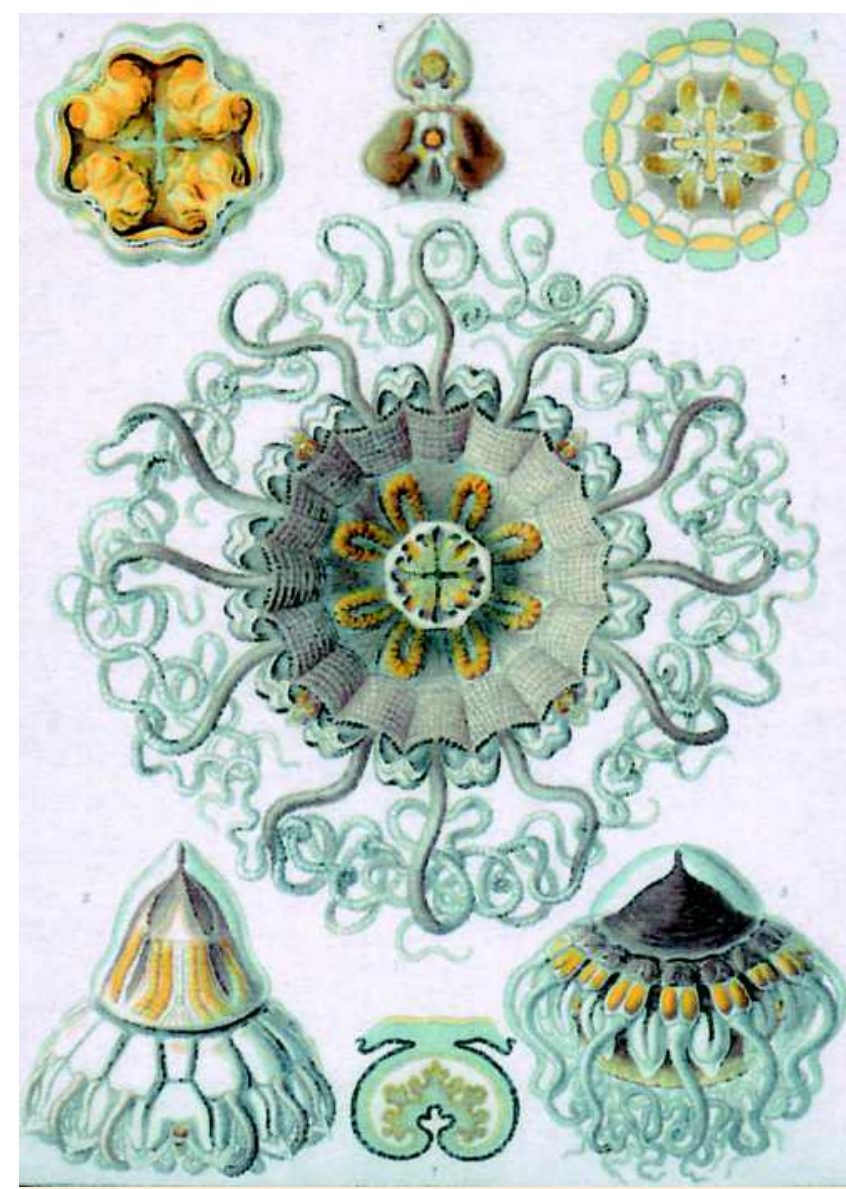

Abb. 4 Medusenqualle (nach Haeckel, E. Kunstformen der Natur. Wiesbaden: Marixverlag, 2004: 91).

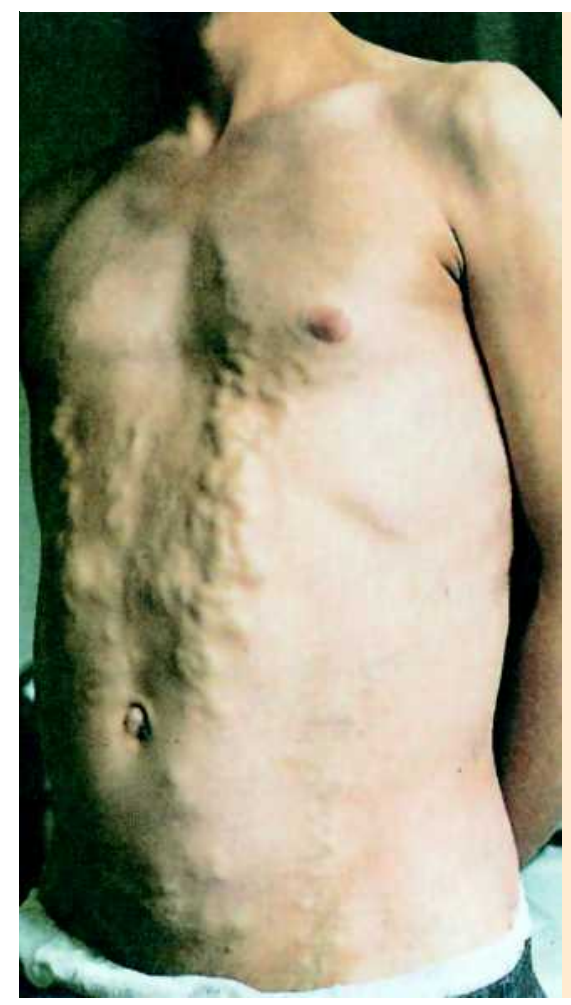

Abb. 5 Das caput medusae der Internisten, schlangenartige Venektasien der vorderen Bauchwand bei etwa infolge Leberzirrhose oder Umgehungskreisläufen (nach Tischendorf, F. W. Der diagnostische Blick, 6. Auflage. Stuttgart: Schattauer, 1998: 167).

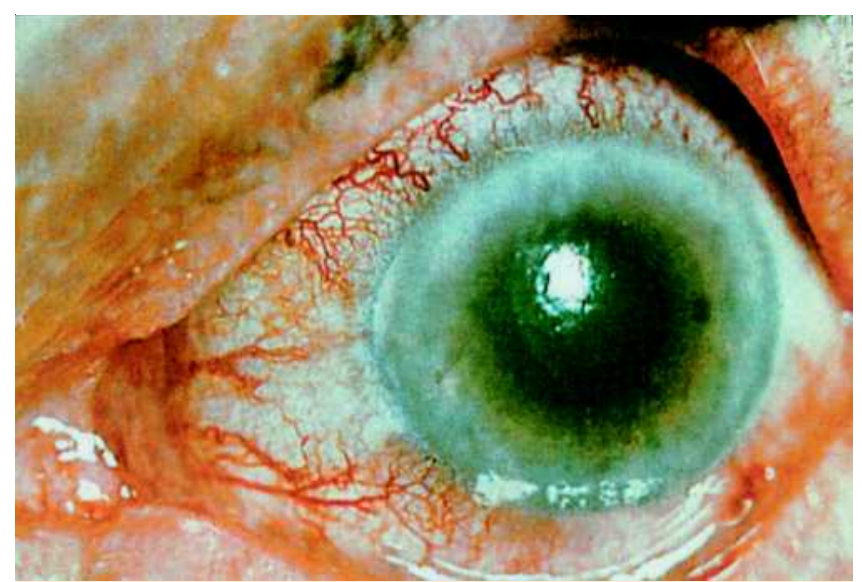

Abb. 6 Das caput medusae der Augenärzte (nach H. J. Küchle, H. Busse und M. Küchle, Atlas der Augenkrankheiten. Bern: Hans Huber, 1999: 102).

\section{Die magisch-mystische Bedeutung von Haar und Zopf $\nabla$}

Ob man auch Haar und Zopf, diesen schon allein wegen der schlangenartigen Form, zu den Ursymbolen zählen will, sei dahingestellt. Einiges spricht dafür. Wer sich näher mit der Geschichte der Haartracht befasst, wird feststellen, dass Haar und Zopf in vielen Kulturen magisch-mystische Qualität beigemessen wurde. Es ist unmöglich, hier auf Einzelheiten einzugehen, deshalb sei nur stichwortartig an Märchen und Mythen (Rapunzel, Pelleas et Melisande), an den ägyptischen Jugendzopf, an die oft durch ein Ritual initiierte Zopftracht bei vielen anderen orientalischen Völkern, Germanen, Galliern, gotischen Priestern, Indianern und Chinesen erinnert. Viele interessante Beispiele und Abbildungen finden sich z. B. bei Corson [3]. portaler Hypertension

\section{Das Medusenhaupt in der biologischen und medizinischen Terminologie $\nabla$}

Was aber hat der Mediziner mit alledem zu tun? Nun, sehr einfach, denn das caput medusae ist auch in die biologische (Medusenqualle, $\bigcirc$ Abb.4) und in die medizinische Terminologie eingegangen. In der inneren Medizin wird darunter die schlangenartige Varizenbildung der Bauchwandvenen, besonders periumbilikal infolge von portaler Hypertension oder venösen Umgehungskreislaufes verstanden ( $\bullet$ Abb. 5). Manchmal wird der Begriff sogar bei auffallenden Kapillarektasien, so z. B. bei entsprechend angeordneten kleinsten Besenreiservarizen der Unterschenkel benutzt. Der Vergleich mit einem Medusenhaupt scheint in beiden Fällen sachlich und optisch weit hergeholt. Dass man ihn trotzdem benutzt hat, zeigt die enorme symbolistische Durchschlagskraft des caput medusae. In der Augenheilkunde ist der Begriff gebräuchlich zur Beschreibung stark gestauter episkleraler Gefäße ( $\bullet$ Abb. 6). Erstaunlich aber, dass er etwa beim Augenhintergrund mit den so gut zu sehenden, ebenfalls schlangenartigen Gefäßen und der Sehnervpapille quasi als Kopf der Medusa keine Verwendung gefunden hat. Wahrscheinlich deshalb, weil nach der Entdeckung des Augenspiegels erst im 19. Jahrhundert und dem rasanten Fortschritt von Technik und Naturwissenschaften Mythos und Mystik zunächst einmal ausgedient hatten, eine Entwicklung, die heute in mancherlei Hinsicht wieder rückläufig zu sein scheint. Sachlich und optisch 


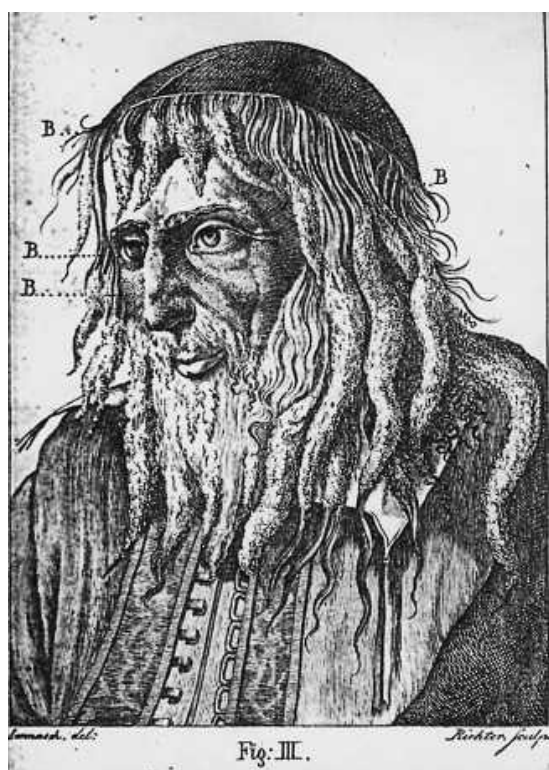

Abb. 7 Weichselzopf vom Typ caput medusae. Hier bei einem anscheinend gut situierten, und nicht krank aussehenden polnischen Juden, vielleicht Rabbi. Offensichtlich artefiziell.

Nach de la Fontaine, der dazu schreibt: „Dieser W. hängt schon an den gesunden Haaren und kann bei „B“ abgeschnitten werden. Da aber die Gesetze dem Juden verbieten, seinen Bart abzuschneiden, so behält er den Weichselzopf so lange, bis er von selbst abfällt." (nach de la Fontaine, FL. Chirurgisch-medizinische Abhandlungen Polen betreffend. Breslau und Leipzig 1792 bey W. G. Korn, 8ov., über den Weichselzopf, 1. Abhandlung).

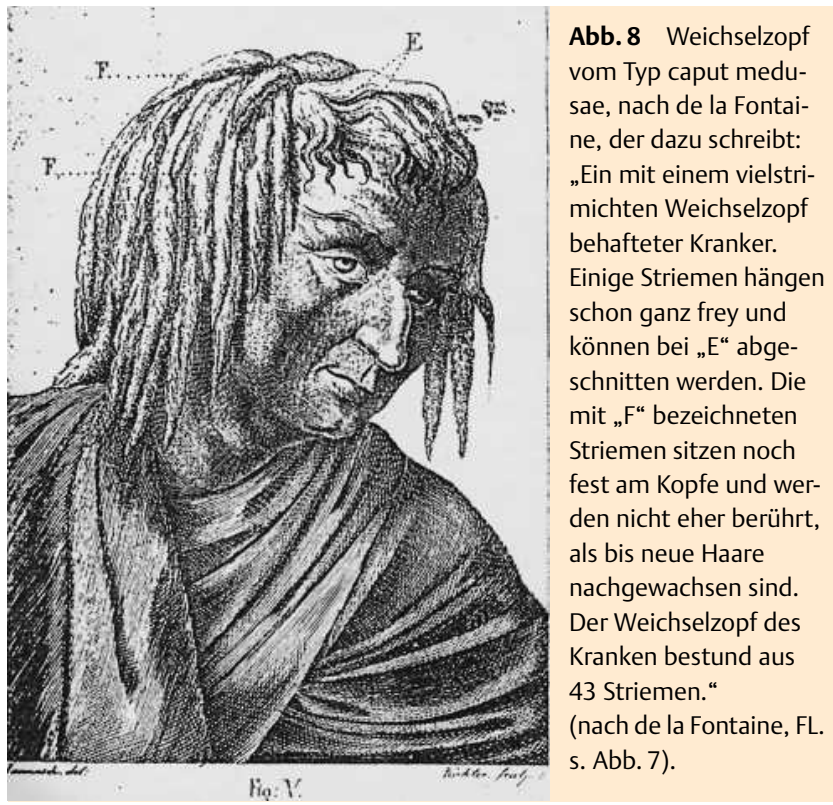

ungleich direkter ist aber die Beziehung des Schlangenhaares zum Caput-medusae Typ des Weichselzopfes (plica polonica), dessen umfassendes Verständnis, wie aus seiner über dreihundertjährigen Medizingeschichte klar wird, nicht ohne die obigen kulturgeschichtlichen Ausführungen zu Zopf und Schlange möglich ist.

\section{Der Weichselzopf vom Typ caput medusae}

In den letzten Jahrzehnten der Weichselzopfära bis zum Anfang des 20. Jahrhunderts verstand man unter der plica polonica nur noch eine extreme Verlausung des Kopfhaares, die durch starken
Juckreiz und Kratzen zu einer Absonderung seröser Flüssigkeit, durch Sekundärinfektion mit Eiter, Krusten und zusätzlich mangelhafter Haarpflege zu einer Verklebung und Verfilzung des Haares in zopfartige Strukturen verschiedenster Formen führte. So z. B. Jesionek [4] im Lehrbuch von Riecke, 1923. Nur am Rande wird noch vermerkt, dass früher weit mehr dahinter steckte, nämlich die sogenannte Weichselzopfkrankheit, bei der es vor allem durch fieberhafte und konsumierende Erkrankungen aller Art, Bettlägerigkeit und hygienische Vernachlässigung akzidentell zur Bildung des Zopfes kam, der in Unkenntnis anderer Krankheitsursachen einfach als Auslöser der Erkrankung angesehen wurde. Läuse waren keineswegs immer im Spiel. Im Zuge der überkommenen Säftelehre glaubte man zudem, dass der Zopf durch eine im Körper vorhandene Dyskrasie als kritisches Produkt entstehe, dabei schlechte Säfte, die sog. Weichselzopfmaterie ausleite, weiterhin dieser kritischen Aufgabe zu dienen habe, und aus diesem Grunde während der aktiven Krankheit und bis zum Nachwachsen gesunden Haares keinesfalls abgeschnitten werden dürfe. Nachdem aber bereits im 17. Jahrhundert und dann vor allem in Frankreich anfangs des 19. Jahrhunderts Gegenstimmen laut geworden waren, die den Zopf für nichts anderes als Aberglauben und Zauberei, allenfalls noch Unreinlichkeit erklärten, kam es ab dem zweiten Drittel des 19. Jahrhunderts im Zuge des allgemeinen Abschiedes von der Säftelehre schließlich zum Ende des Weichselzopfes als eigenständiger Krankheit. Bauer, der sich mit dieser Zeit des Umbruchs und der Entwicklung hin zur naturwissenschaftlich exakten Medizin aus medizinhistorischer Sicht besonders beschäftigt hat, schildert in einem Beitrag in dieser Zeitschrift [5], wie es auf Kongressen der deutschen Naturforscher und Ärzte von 1838-1868 zu letzten kontroversen Diskussionen zwischen Krankheitsverfechtern und -leugnern kam. Zu den entschiedensten Krankheitsgegnern, die sich langsam aber sicher durchsetzten, gehörte der Direktor der Hautklinik an der Charité, v. Baerensprung, der auf dem Kongress in Bonn 1857 klar und eindeutig erklärte, dass der Weichselzopf nur das Produkt eines eingewurzelten Vorurteils sei.

Doch zurück in die Blütezeit des Zopfes, ins 17. und 18. Jahrhundert. Glaubte man an die Plica als gefährliche Krankheit, so musste ihr Auftreten dennoch ambivalent beurteilt werden. Einerseits war diese durch finstere dämonische Mächte bewirkte Haarverwirrung ein für alle sichtbarer schwerer Schicksalsschlag, verbunden mit vielem wirklichen oder eingebildetem Leiden, das mit ärztlicher Hilfe und oft genug auch mit Beistand eines zopfkundigen Geistlichen geduldig ertragen werden musste. Andererseits war der Zopf aber wegen der Ausleitung schlechter Säfte gleichzeitig ein günstiges Zeichen, ja ein Heilmittel. Je größer und prächtiger der Zopf, desto besser die Prognose. Nur folgerichtig, dass man in einer weiteren Phase der Weichselzopfära auf die Idee kam, sich eine artefizielle Plica zur Therapie und sogar zur Prophylaxe aller möglichen Krankheiten zuzulegen. So lassen denn auch die meisten Abbildungen aus dem 18. Jahrhundert ganz sicher eine artefizielle Verursachung vermuten. Äußerlich nahm man dabei u.a. Pech, Harz, Wachs und Fäden zu Hilfe, und die ganz Schlauen versuchten sogar systemisch mit teilweise widerwärtigen Methoden, so z.B. Trinken von alkoholischen Tinkturen, in denen vorher eine alte Plica eingeweicht worden war, den Zopf zum Wachsen zu bringen. Es kamen dabei die verschiedensten Formen heraus, von denen der Typ caput medusae ( $\bullet$ Abb. 7 und $\bullet$ Abb.8) mit seiner Minimalvariante coma caesarea zu den häufigsten zählte. 


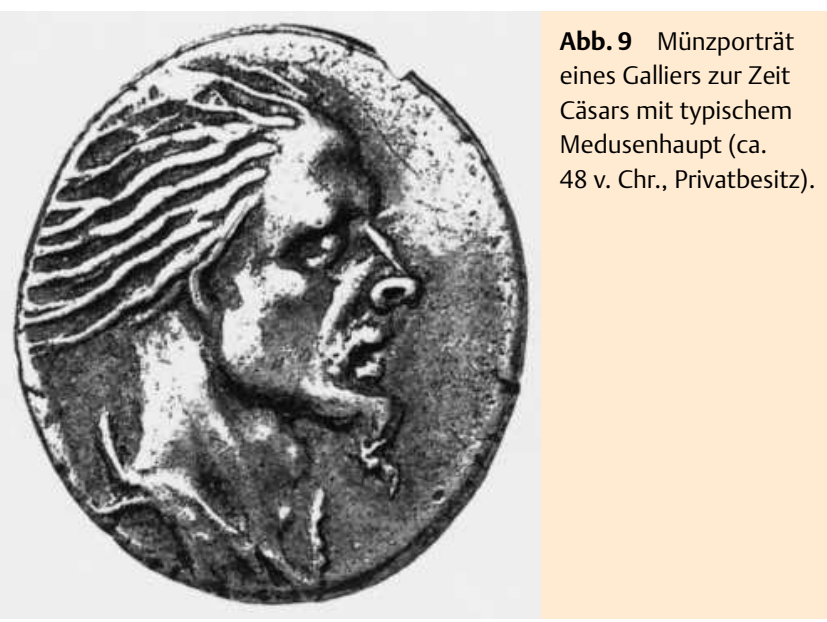

Erstbeschreibung zwischen 1580 und 1600 $\nabla$

Die ersten medizinhistorischen Erwähnungen des caput medusae im Zusammenhang mit dem Weichselzopf stammen aus der Zeit um 1580-1600. Zuerst lesen wir bei Grafenberg [6] (1584): „Horridum quoddam impexum adeoque intricatum capitis atque barbae capillitium apud nostros haud infrequens, ceterum veteribus cuiuscunque aetatis medicis incognitum observare licet, quo affecti praelongas capillorum tricas et cincinnos mirifice intricatos digiti saepe crassitie ex reliquo capitis et barbae capillitio ad humeros, pectus et aliquando ad umbilicum usque demissos propendere videas, aspectu plane horrifico et Gorgoneum caput praeferente. "Deutsch: Man kann die recht ungekämmten und deutlich verwirrten Haare des Kopfes und des Bartes bei uns (das bedeutet in diesem Fall in der Rheingegend, d. Verf.) nicht sehr selten beobachten, übrigens den alten Ärzten dieser Zeit noch unbekannt, wobei die sehr langen Haarflechten und Locken, sonderbar verworren und oft fingerdick aus den übrigen Kopf- und Barthaaren auf die Oberarme, die Brust und manchmal bis zum Nabel herabzuhängen scheinen, ein ganz schrecklicher Anblick, wie bei einem Gorgonenhaupt.

Das caput medusae ist danach in der äußerst umfangreichen Literatur über die Plica bis ins 20. Jahrhundert allgemein üblicher Sprachgebrauch.

Hercules Saxoniae (Ercole Sassonia), Medizinprofessor zu Padua (1551 - 1607) [7] geht in seiner Schrift über die Plica sogar noch einen Schritt weiter und entmythologisiert gleichsam das Medusenhaupt der Antike, indem er die These vertritt, dass dieses eine Darstellung multipler Weichselzöpfe bei wirklichen Menschen gewesen sei. „Caput Gorgoneum, caput furiarum vera humana capita fuisse, et fictitis poetarum occasionem praebuisse." Deutsch: Gorgonen- und Furienhaupt sind wahre menschliche Häupter gewesen und boten den Vorstellungen der Poeten (gute) Gelegenheit. Wenn also Hercules Saxoniae den Weichselzopf schon in der Antike für möglich hält, dann wäre auch sein Vorkommen im Mittelalter nicht verwunderlich, und der Künstler in Spieskappel hat vielleicht Kenntnis davon gehabt. Er hat dann die Plica zwar nicht in allen naturalistischen Einzelheiten dargestellt, aber die zugrunde liegende Vorstellung dämonischer Haarverwirrung in allegorisierender Weise und vielleicht überlagert mit anderer Symbolik ausdrücken wollen. Das Münzporträt eines Galliers zur Zeit Cäsars ( $\bullet$ Abb. 9) zeigt in erstaunlicher Detailtreue ebenfalls eine Zopftracht, die dem Medusenhaupt kaum nachsteht. Auch hier ist man in Versuchung, eine frühe Darstellung des Weichselzopfes anzunehmen. Die Autoren

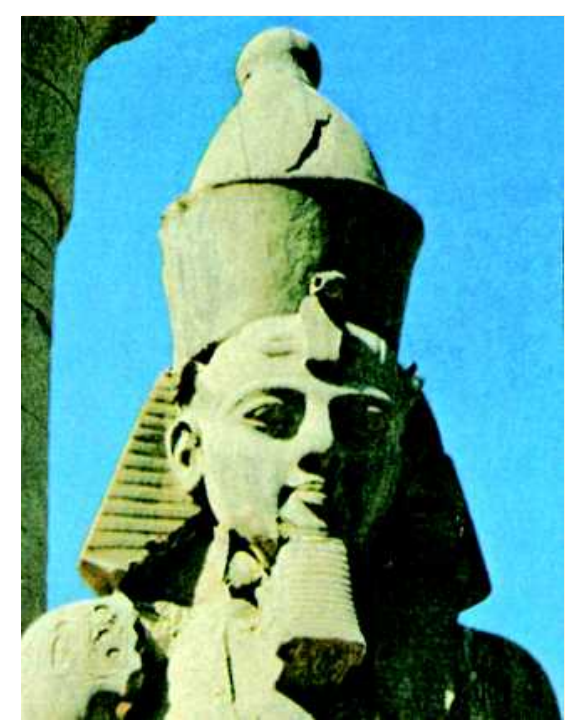

Abb. 10 Pharaonenkrone, von einer Königsstatue am Karnak-Tempel (nach Strelocke, H. DuMont Kunst-Reiseführer Ägypten, Farbtafel 21. Ostfildern: Mairdumont/ Falk-Verlag, 1979).

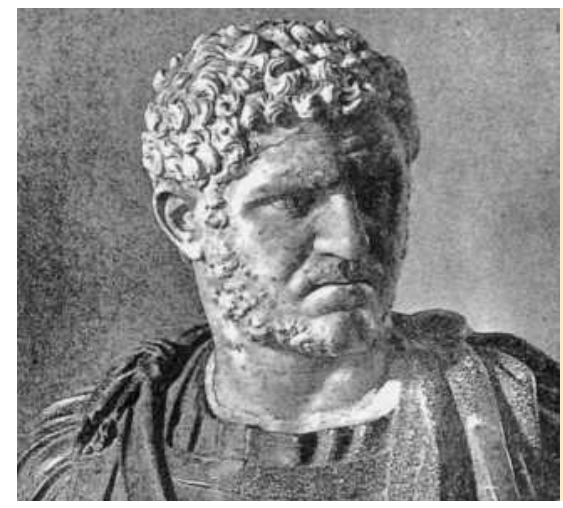

Abb. 11 Coma caesarea, hier beim römischen Kaiser Caracalla. Die Lockenpracht soll Überlegenheit und Machtanspruch ausdrücken, Rom, Kapitolinisches Museum.

aus dem 16. Jahrhundert beschreiben die Plica zwar erst für die Zeit etwa ab 1570, halten aber ausdrücklich auch ein früheres Auftreten für möglich.

\section{Außermedizinische Gründe für den artefiziellen Weichselzopf \\ $\nabla$}

Der Begriff Weichselzopf (trotz besonders häufigen Vorkommens in Polen, das andere Ursachen hat, kein Zusammenhang mit dem Fluss Weichsel) syn. Wichtelzopf, Hexen-Trudenzopf, Mährenflechte, Alpschwanz u.v.a. - ähnliche Terminologie in vielen anderen Sprachen - soll sich vom polnischen Wiescy$\mathrm{ca}=$ Hexe ableiten und zeigt ohne weiteres, dass es außer den medizinischen noch andere Gründe gab, sich einen artefiziellen Weichselzopf, speziell vom Typ caput medusae zu verschaffen. Durch pseudoreligiöse Vorstellungen uralten Zopfrituals, durch abergläubische Magie und Zauberei soll mithilfe des archaischen Ursymbols Schlangenhaar autoritäre Dominanz und überlegener Machtanspruch ausgeübt werden, der wie das mythologische Vorbild einen respektheischenden, erschreckenden und quasi versteinernden Effekt auf die Menschen bewirkt, besonders wichtig z.B. auch bei der Kriegsführung, wie die o.g. Abb. des gallischen Kriegers ohne weiteres nahe legt. Sehr eindrucksvoll auch die furchterregende Uräusschlange an der ägyptischen Pharaonenkrone ( $\bullet$ Abb. 10), und die ebenfalls analog in der Weichselzopfterminologie vorkommende „coma caesarea“ antiker Machtmenschen ( $\bullet$ Abb. 11), die aber bestimmt auch nicht 
immer so reichliche, natürliche Locken hatten und häufig Wachs, Pech und Fäden zu Hilfe nehmen mussten. Bei den Machtmenschen des Absolutismus in der Barock- und Rokokozeit hat dann die Perücke eine entsprechende Ersatzfunktion für die natürliche Haarpracht ausgeübt. Gleichzeitig aber war es die Blütezeit des Weichselzopfes, der zwar gerade beim polnischen Adel keineswegs besonders selten war, im Großen und Ganzen aber doch sozusagen als Perücke des kleinen Mannes ein Attribut der sozialen Unterschicht geblieben ist.

\section{Das „Kollektive Unbewusste“ - eine Brïcke zur ganzheitlichen Betrachtung}

Wir haben gesehen, dass das archaische Ursymbol Schlange in Verbindung mit einem ebenfalls in vielen Kulturen praktizierten magisch-mystischen Zopfkult eine gemeinsame Basis beim Verständnis von mythologischem Medusenhaupt, mittelalterlichem Schlangenhaar und auch beim Medusenhaupt der Mediziner ist. Eine Brücke dazu baut zusätzlich die Lehre C. G. Jungs vom „kollektiven Unbewussten“ [8]. Demnach gibt es bei allen Menschen neben dem „persönlichen Unbewussten“ die noch tiefere seelische Schicht des „kollektiven Unbewussten“, weil dieses „nicht individueller, sondern allgemeiner Natur ist, das heißt, es hat im Gegensatz zur persönlichen Psyche Inhalte und Verhaltensweisen, welche überall und in allen Individuen cum grano salis die gleichen sind. Es ist, mit anderen Worten, in allen Menschen mit sich selbst identisch und bildet damit eine in jedermann vorhandene, allgemeine seelische Grundlage überpersönlicher Natur, die Inhalte des kollektiven Unbewussten sind die so genannten Archetypen.“ (S. 77/78). Die Schlange ist im Jungschen Sinn selbst zwar kein Archetyp. Aber neben den Drachen sind „Schlangen und andere niedere Vertebraten seit alters beliebte Symbole der kollektiven psychischen Grundlage“ (S.190). Diese ist demnach allen Kulturen und Zeiten gemeinsam, woraus sich ergibt, dass sie bei vielen Menschen auch ohne direkte Kenntnis voneinander immer wieder in Religion, Aberglauben, Kunst, Mythologie und sogar Wissenschaft und Medizin zum Ausdruck kommt.

\section{Abstract}

\section{The Head of Medusa in Mythology and Medicine - Attempt at a Holistic Approach \\ $\nabla$}

The depiction of snake hair on a medieval capital was the reason for establishing a relationship between the caput medusae of Greek mythology and the notion which appears at least three times in medical terminology. There is a particularly close correlation with a caput medusae-variant known from medical history: the elflock. This elflock, especially in its artificial form, also has a magical and mystical quality, just as its ancient model of the head of Medusa. With the help of C. G. Jung's concept of the "collective unconscious" and the prime symbol of the snake rooted in it, an attempt will be made at a holistic approach of all manifestations of the caput medusae in mythology, art, culture, biology and medicine.

\section{Literatur}

1 Jost G (Hrsg). Stille, Licht und Klarheit: Kirchen in Kurhessen-Waldeck. Kassel: Verlag Evangelischer Medienverband, 2004: 88

2 Michler W. Kloster Wienhausen, die Wandmalereien im Nonnenchor. Klosterführer. Kloster Wienhausen, 1968: 56

3 Corson R. Fashions in Hair (the first thousand years). London: Peter Owen, 1965, dort zahlreiche Abbildungen

4 Jesionek A. Kapitel I, Teil II. In: Riecke E. (Hrsg). Lehrbuch der Hautund Geschlechtskrankheiten, 7. Auflage. Jena: Gustav Fischer Verlag, 1923: 523

5 Bauer AW. Der „Weichselzopf“ in medizinhistorischer Perspektive. Eigenständige Hautkrankheit oder mythologisches Konstrukt. Akt Dermatol 2004; 30: $218-222$

6 Grafenberg Johannis Schenckii. Observationem medic.rarorum, lib. VII, lib I de capite, observ XII de tricis incuborum, Basileae, 1584. Zitiert nach: Butzke EL. Denkschrift über den Weichselzopf. Ein Beitrag zur Begründung einer rationellen Pathologie und Therapie desselben. Thorn: Commissionsverlag von Ernst Lambeck, 1858: 168

7 Hercules Saxoniae Patavini. De plica, quam Poloni Gwozdziec, Roxolani Koltunum vocant. Patavii: 1600: 4

8 Jung CG. Archetyp und Unbewusstes. In: Barz H. et al. (Hrsg). Grundwerk C. G. Jung, Band 2, 5. Auflage. Zürich und Düsseldorf: WalterVerlag, 1996: 77-78, 190 